\title{
Space Weather Monitoring from Observatorio Astronómico of the Universidad Tecnológica de Pereira
}

\author{
Galvis H. D, Galeano D, Quintero E. A \\ Universidad Tecnológica de Pereira, Pereira, Colombia \\ Email: (hdgalvis@utp.edu.co, david.galeano@utp.edu.co, equintero@utp.edu.co)
}

Accepted : 27 January 2020

\begin{abstract}
The only space weather monitor located in Colombia, that at the present time continuously reports on X-ray solar activity, is the monitor that belongs to the Research Group Astroingeniería Alfa Orión. This device is officially registered as UTP 0383 in the Stanford Solar Center server and is located at the Observatorio Astronómico de la Universidad Tecnológica (OAUTP). The monitor has allowed the study of solar activity since 2013, due to the implementation of an electronic system specially designed to work at the frequency of $23.9 \mathrm{kHz}$, which better suits the local conditions. The solar records and detections are obtained through indirect measurements of the NAA, in which the solar X-ray behavior can be observed. Currently, the OAUTP holds a database that is the result of 5 years of observations, and contains confirmed detections of solar flares of classes $C, M$, and $X$. The infrastructure developed in radioastronomy at the OAUTP have allowed a deeper research of the celestial bodies in the west of Colombia. This is a differentiating factor in relation to other national astronomical observatories that work with radioastronomy and space weather. Finally, the Grupo de Investigación Alfa Orión has contributed significantly to the study of space weather from Colombia by registering and publishing their records in international databases.
\end{abstract}

(c) 2019 BBSCS RN SWS. All rights reserved

Keywords: Radioastronomy, spaceweather, solar flares.

\section{INTRODUCTION}

The ionosphere, a dynamic system in constant transformation located between 80 and $500 \mathrm{~km}$ from the sea level, is used in communication, effective transmission of information, and realtime data acquisition, through the propagation of electromagnetic waves (EMW) that are reflected off from ground sources and return to Earth in a specific place. Changes in the atmosphere, produced by EMW from celestial bodies or variations in Earth's electromagnetic field, alter the behavior of this zone, with consequences in communication systems such as maritime, GPS, and aerospace systems. These variations are, for the most part, due to X-ray solar flares or to coronal mass ejections, which send enormous quantities of charged particles and radiation into the interplanetary space (Quintero et al., 2014). The radiation travels to Earth and interact with the ionosphere, disturbing its electrical properties, causing radio blackouts and damage to satellite equipment. Space weather monitoring is the study of the effects caused by the Sun and its interaction with the ionosphere (Jaramillo et al., 2017).

The Space weather monitoring takes place using the very low frequency band (VLF), located between 3 and $30 \mathrm{kHz}$. The signals that are recorded in this bandwidth allow the interpretation of the density and location changes of the ionospheric layers (parameters that vary due to stimulation caused by solar radiation) since the emitted signal bounces off in the layer closest to the Earth (Manoj and Sundaram, 2016). Different research centers around the world have been recording the signals emitted by VLF stations, developing, for example, studies around the effects of solar radiation on space operations (Shea and Smart, 1998), predicting dynamic behavior of the ionosphere (Petry et al., 2014) or the optimization of VLF receptors to record disturbances associated with solar glare (Kulkarni et al., 2008). Moreover, the VLF stations have also been used to solve astrophysical problems, e.g., the monitoring of cosmic explosions (Tanaka et al., 2010; Inan et al., 2007), or the detection of the ionosphere response to natural phenomena as the seismic activity (Grant et al., 2015). Most of these research centers send their observations to the Stanford Solar Center (SSC), where they are stored in a global, free-access database. However, it is important to say that there exist other VLF networks that have contributed significantly to the study of the space weather, such as the SAVNET network (Raulin et al.,
2009; Raulin et al., 2010) that operates in South America, or the work done in India with VLF networks that use VTX transmitters (Sasmal et al., 2014; Chakraborty et al., 2017).

Space weather monitors are VLF wave receivers located around the world that are responsible for indirectly recording the behavior of the ionosphere. Indeed, these devices observe terrestrial radio sources, which modify their waveform due to the X-ray emissions from the Sun. With the implementation of these monitors, signals emitted by earth-based transmitters, such as NML, NPM, NLK, etc, can be registered. Because of its power and signal quality, one of the most important broadcasters is the NAA station, located in Cutler, Maine USA. It is used by the US naval for transatlantic submarine communication (Quintero et al., 2014). This station emits with a power of $1 \mathrm{MW}$ in the frequency of $24 \mathrm{kHz}$, and its waveform is such that abrupt changes, due to the X-rays solar activity, can be clearly identified.

Space weather monitoring is a global issue, worth of scientific interest and as such, it demands international and collaborative efforts in order to create a network that allows the study of the space weather (Ammar and Ghalila, 2016). Unfortunately, up to this date, Colombia did not participate in this global network. Therefore, the aim of this project was to develop and implement a space weather monitor in the Observatorio Astronómico of the Universidad Tecnológica de Pereira (OAUTP), that participates in this international network, by sending data that strengthen the international scientific cooperation. This paper is the compilation of what has been the development, implementation, and tuning of a space weather monitor with the appropriate characteristics to record the NAA signal and track X-ray solar activity. This project began in 2013 at OAUTP. Nowadays, it is an integral system registered in the SSC data base with UTP code 0383. It has a receiver designed for local electromagnetic characteristics, a custom implemented antenna, and a real-time data acquisition and visualization system that loads all the online information.

\section{MATERIALS AND METHODS}

The SSC space weather monitoring program developed two VLF receivers that were distributed among the educational and scientific communities with the aim of creating an international network dedicated to observing the solar behavior. One of these, called the Sudden Ionospheric Disturbances (SID) receiver, is a 
simple, low-cost device that registers the amplitude of the variations in the Ionosphere behavior, in a user-selected frequency. The other device, the Atmospheric Weather Electromagnetic System for Observation Modeling and Education (AWESOME) monitor, is a professional receiver with a larger sensitivity, bandwidth and cost than the SID receiver. AWESOME can simultaneously register a VLF frequency spectrum both in amplitude and phase. Here, a monitor based on the SID architecture was selected, as it is a simpler and considerably cheaper device than the AWESOME monitor, which makes it more viable to introduce and spread within the Colombian scientific community. Hence, the OAUTP monitor is tied to the SSC SID network.

The SID system of the Universidad Tecnológica de Pereira (SID UTP) is a radio telescope for the observation and recording of VLF waves emitted by the NAA station, selected for its location and emission power. This radio telescope has a square antenna of $2 \mathrm{~m}$ diagonal, a radio receiver responsible for filtering the signals at $24 \mathrm{kHz}$ and computer equipment. Once the antenna captures the signal, the receiver performs the electronic acquisition and preprocessing. Then the digital processing is performed through the implementation of a software designed for this specific task, which allows us to capture and store the data locally and remotely. Finally, it is possible to visualize the solar behavior in real-time from the server, in addition to having access to the graphics stored in the remote database. The implementation of the space weather monitoring system was developed based on three aspects: antenna (Quintero et al., 2014), radio receiver (Jaramillo et al., 2017), software and database. Each of these is described more specifically below.

\subsection{Loop Antenna}

A square loop antenna was built with a response in the band $24 \mathrm{kHz}$, to capture and transduce the signal from NAA in a voltage. As shown in (Quintero et al., 2014), the antenna design geometry corresponds to a square structure with $2 \mathrm{~m}$ edges, in which $100 \mathrm{~m}$ of insulated cable is wrapped. After the acquisition and transduction, an average voltage signal of $200 \mathrm{mV}$ is observed at the BNC terminal of the coaxial, which will be taken to the receiver for the next stage. Figure 1 shows the antenna installed at OAUTP.

\subsection{Radio Receiver}

The SID radio-receiver is designed to be distributed around the world. These devices detect the different VLF stations thanks to their large bandwidth, and the frequency can be set digitally. Given the local conditions, the best signal-to-noise ratio was obtained by tuning our device towards the NAA station. For this reason, for the conditioning of the signal, there is a narrow-band radio receiver of own construction, centered at $24 \mathrm{kHz}$, providing a maximum power of $56 \mathrm{~dB}$ to the signals that meet the frequency condition. A sixth-order bandpass filter was designed to achieve the best level of selectivity, with a maximum flat response (see fig. 2). In order to simplify the analog construction of the filter, a method was implemented that consists of reducing the order of the system by decomposing the transfer function into simpler subsystems (Jaramillo et al., 2017). This methodology allows implementing a higher-order filter in banks of smaller order filters based on operational amplifiers. Figure 3 shows the assembly where the linear combination of the response of each subsystem reconstructs the given system.

As a final step, the output terminal of the filter is taken to the auxiliary input of a computer's sound card, performing the acquisition and digitization of the signal with a sampling rate of $96 \mathrm{kbps}$ for further processing and storage.

\subsection{Database}

In order to provide local data to the international collaboration that promotes space weather research, the information acquired by the OAUTP system is processed by the SSC SuperSID program that allows the capture, visualization, and storage of the received data, updating the information online every 24 hours. Next, in Figure 4, the SSC platform is observed with one of the recorded signals in which X-ray activity is evidenced.

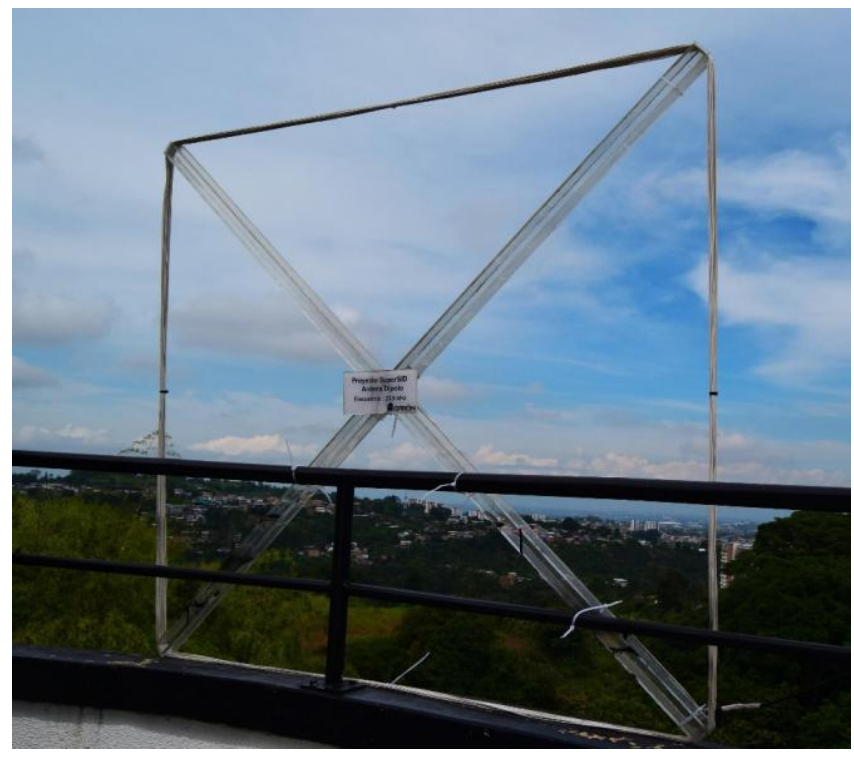

Figure 1. Rectangular dipole antenna with $2 \mathrm{~m}$. of diagonal, $100 \mathrm{~m}$. of 22 AWG cable, and ending in a coaxial of $75 \Omega$.

With the intention of improving the performance of the space weather monitoring system at OAUTP, the DSOA software was developed, focused on the detection, mathematic processing, and storage of the signals coming from the radio telescope in a local database. The most notable characteristic of this software is the Web interface that makes it possible to visualize the gathered data together with the previously stored data from the remote data base.

In the block diagram of Figure 5, the software operating cycle is shown. In the first stage, the capture of information from the radio telescope through the computer's HD sound card begins. The opening and configuration of the sound port parameters are carried out from the screens containing the designed application. In the second stage, the spectral density of the signal is determined through the Welch method. This method is complemented by Hann's winding method, determining the frequencies contained in the signal when analyzing overlapping segments of this (Quintero et. al, 2014). In this process, the central frequency of the Welch filter is defined for the capture of signals at $24 \mathrm{kHz}$. In the third stage, the electronic noise generated by the audio port is eliminated, implementing an average recursive filter. The fourth and penultimate stage constitutes the storage of the data captured by the application in a local database, guaranteeing the consultations from the web page in the final phase. Figure 6 shows the graphic environment of the application developed by the OAUTP. The window to start data collection is displayed by clicking on the Capture button. Among the options available, the Consult Spectrum button accesses the graphic display of the frequency distribution contained in the signal. On the other hand, through the Configuration button, it is possible to view and modify the operating parameters of the application, allowing them to adjust the frequency of interest. 


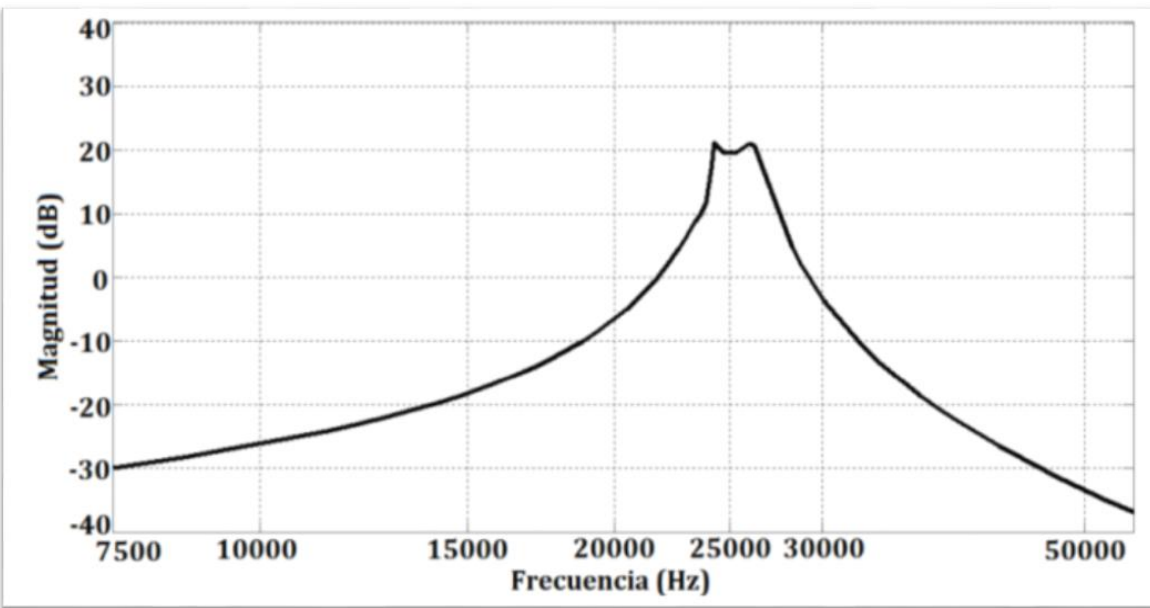

Figure 2. Response in frequency of the sixth-order passband Butterworth filter designed for OAUTP.

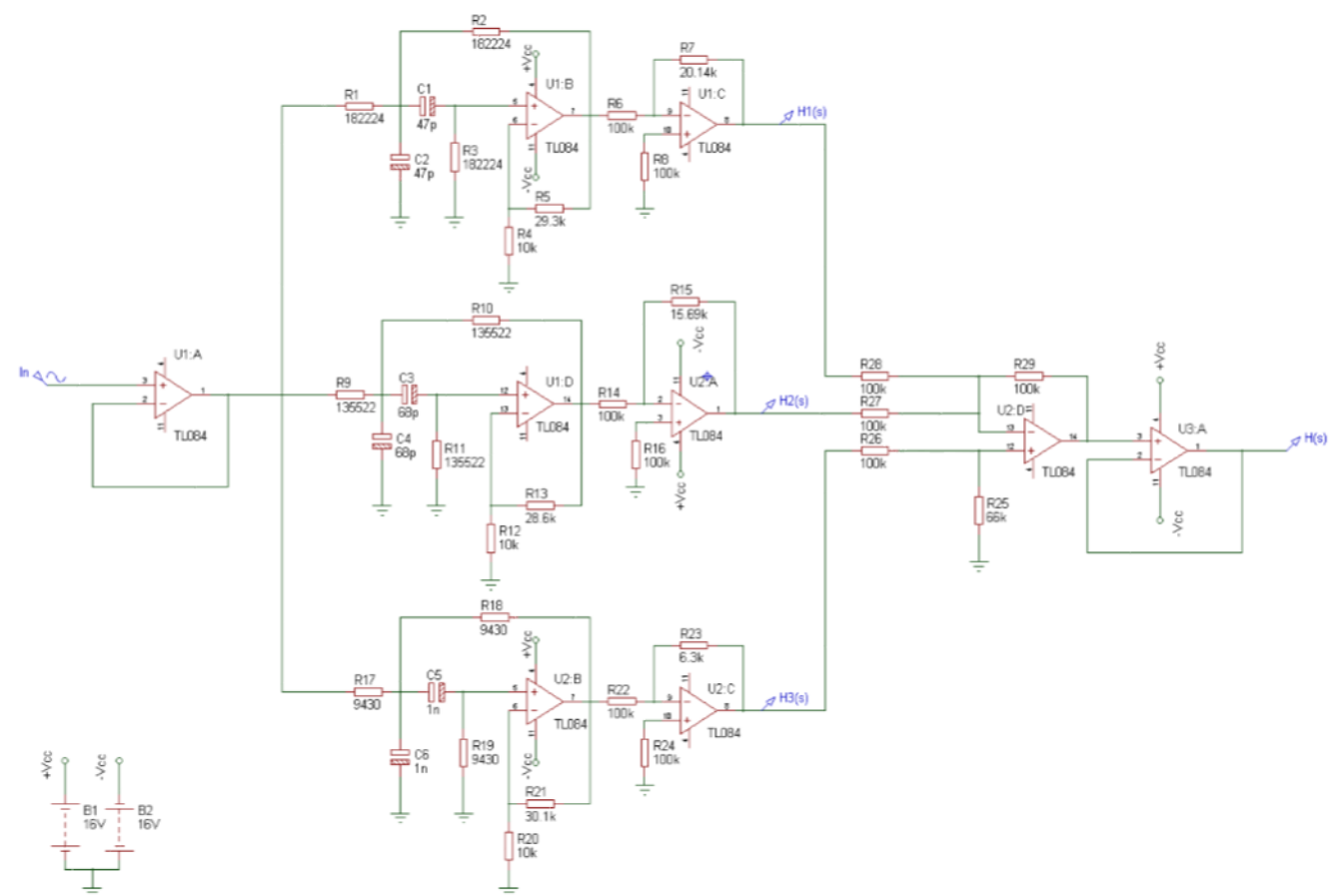

Figure 3. Diagram of the receiver schematic of central frequency waves $23.9 \mathrm{kHz}$ and gain of $56 \mathrm{~dB}$. 


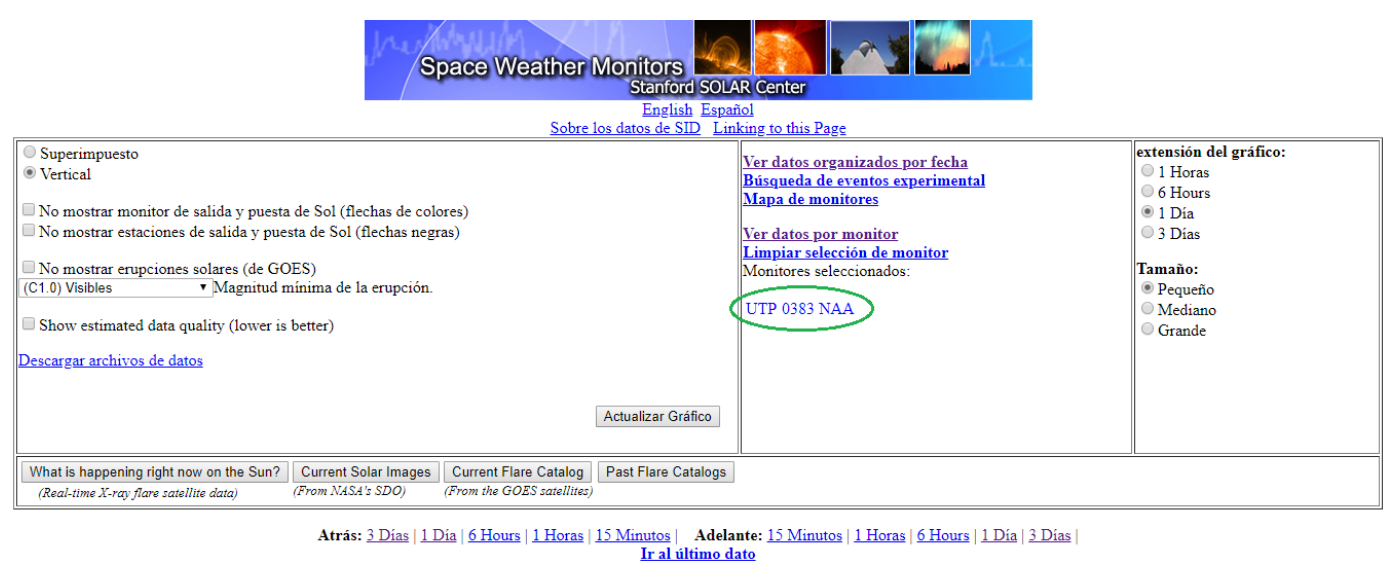

El gráfico comienza en: 26 de enero de 2019 12:00:00 a.m. GMT

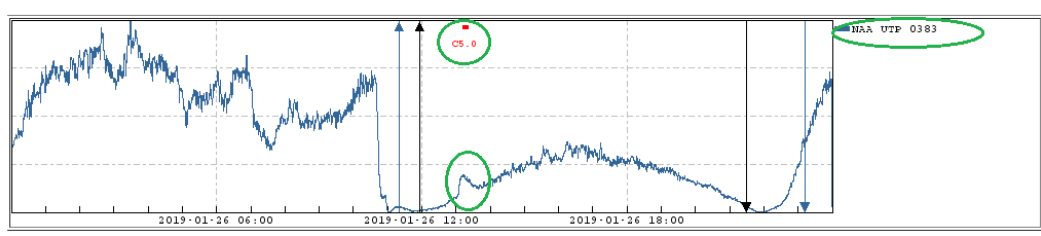

Figure 4. Stanford Solar Center's database for the monitoring of space weather with the UTP 0383 signal and the detection of a C5.0 Xray flare encircled in green.

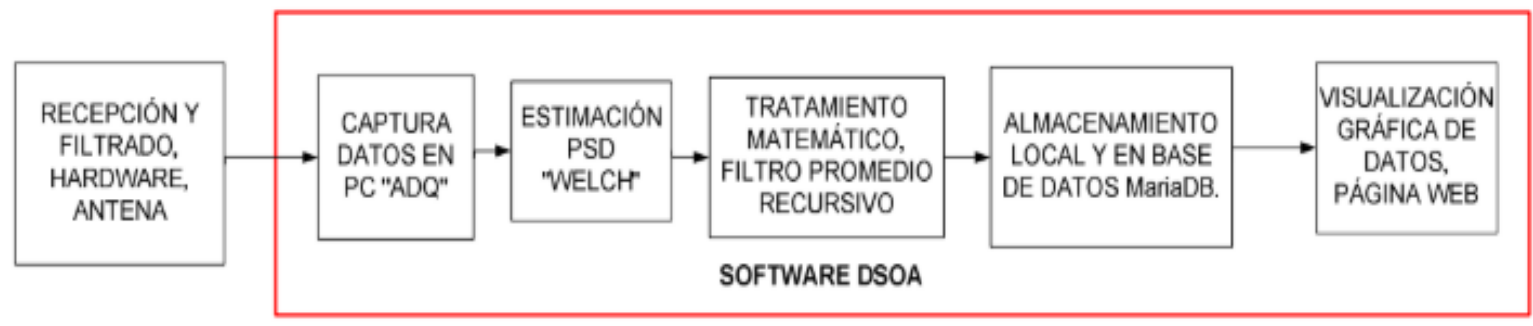

Figure 5. Flowchart of the DSOA software.

Additionally, the archival of data obtained at OAUTP can be downloaded from the SSC or from DOSA. The local database can be accessed from our web application (http://observatorioenlinea.utp.edu.co/ssid/), while the remote database can be reached through the SSC server (http://sid.stanford.edu/database-browser/) .

\section{RESULTS}

The space weather monitor at OAUTP monitors the NAA signal (1MW power, $\mathrm{f}=23.9 \mathrm{kHz}$, Cutler, Maine USA). The system has been operational since 2013. During this time, it has recorded 531 solar flares $(126 \mathrm{C}, 11 \mathrm{M}$ and $11 \mathrm{X})$. Figure 7 show the history of the most significant X-ray flares that have been registered by the OAUTP space weather monitoring system. For each one of the signals, the horizontal axis indicates the time (for 24 hours and with 5 seconds of sampling), which allows to identify the initial and final moments of the detection. The vertical axis is an indirect measure of the signal intensity, as given by the receiver electric response. The changes in intensity allow one to identify and correlate the X-ray events. Currently, our device measures only the amplitude of the signal. As future work, the intensity data can be calibrated as done in Sharma and More (2017) in order to measure directly in volts.

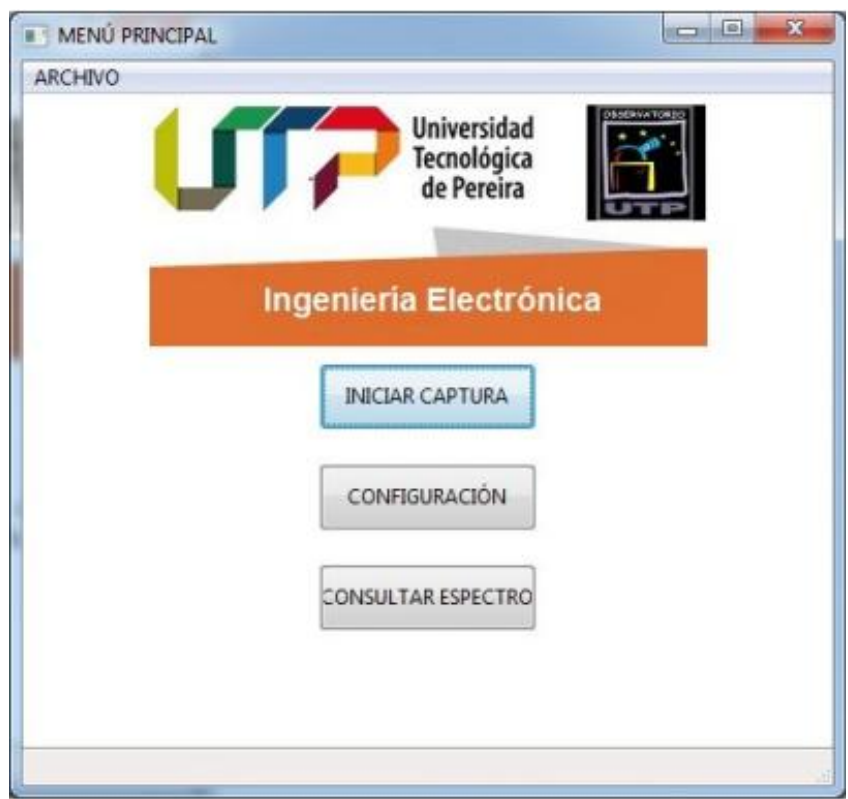

Figure 6. Main menu of the DSOA software. 

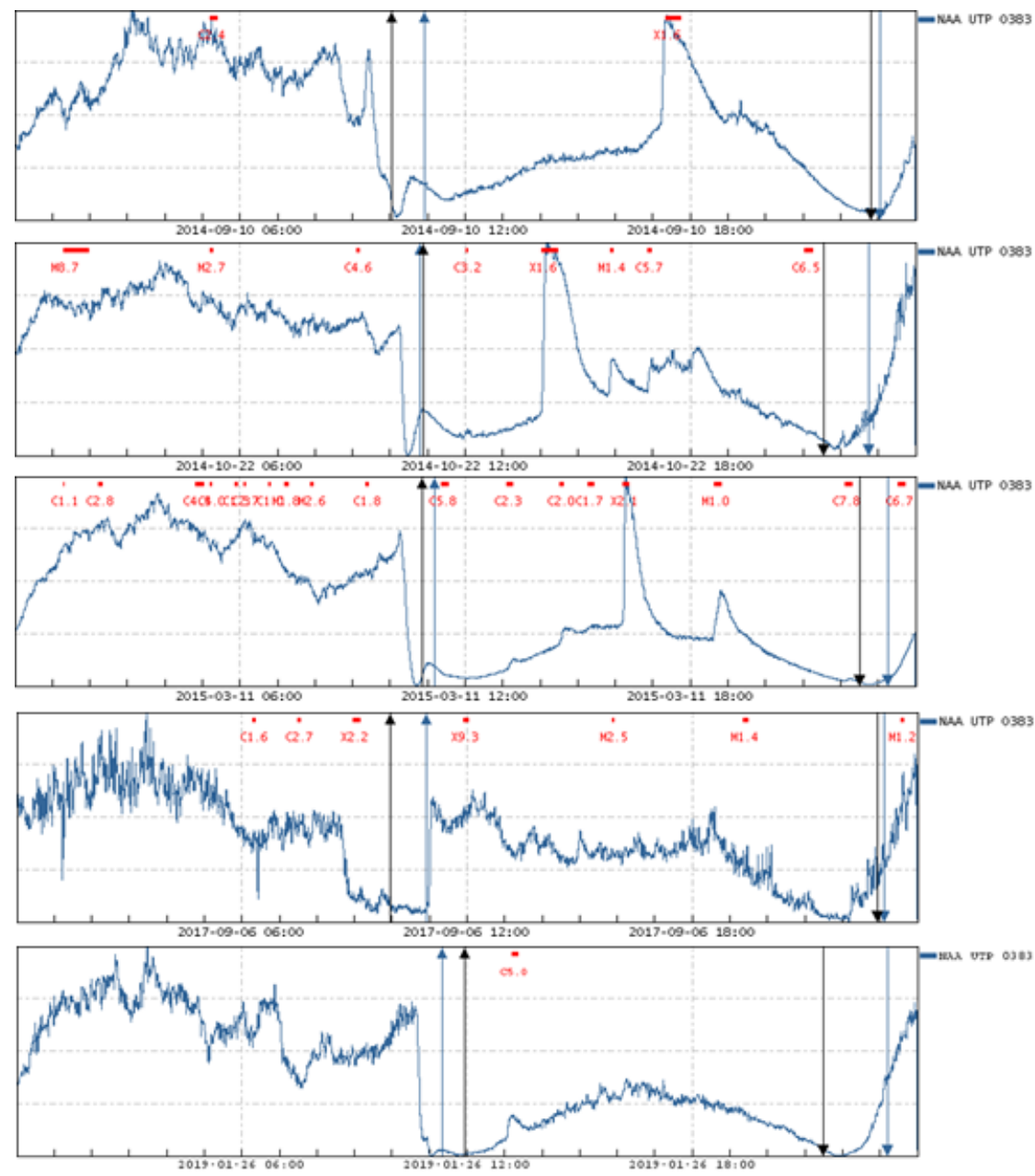

Figure 7. Solar flares detected by OAUTP monitor from September 2014 to January 2019. 


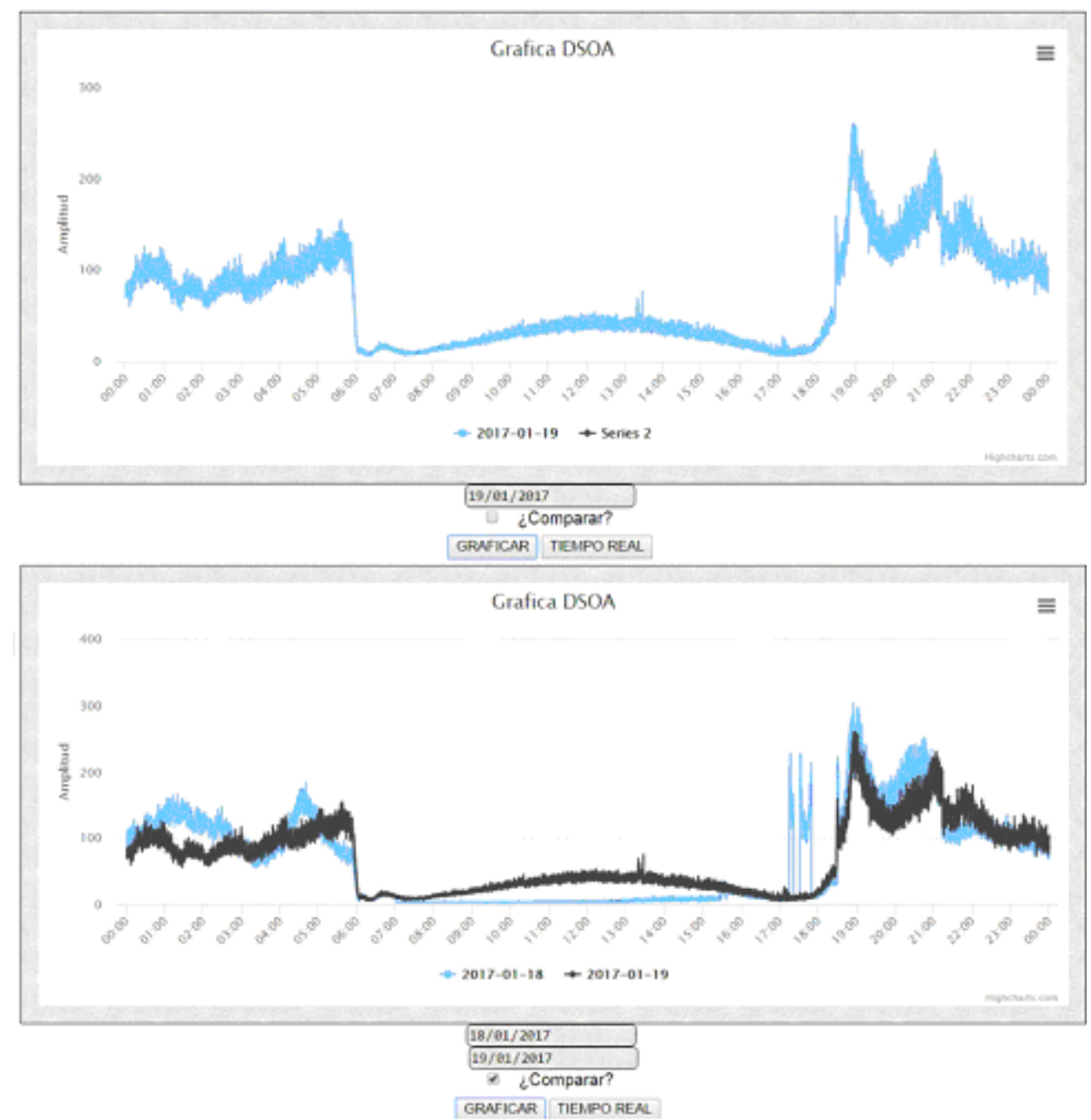

Figure 8. Database of the space weather monitoring system in Colombia developed by OAUTP.

Moreover, the software developed by OAUTP allows a better interpretation of the information obtained, since the acquisition and processing of the data are done in parallel. The Web application guarantees a real-time visualization as well as the visualization of historical data that have been previously stored by the DSOA software or included manually, as shown in Figure 8. It also facilitates the comparison between the said historical data, allowing the visualization of substantial differences in the behavior of space weather during different dates.

The system operates efficiently, as shown by the fact that other researchers use the OAUTP data as point of comparison for their results (see Fig. 9). The developed web app, DSOA, makes easier the comparison and real-time visualization of the obtained data, and this allows a better interpretation of the space weather behavior. This project has raised the interest of the general public in solar phenomena and for this reason, scientific press releases have been written about space weather and its effects on Earth (see Figure 10).

\section{CONCLUSIONS}

The 5 years of data obtained by the SID UTP receiver enrich the global database of the Stanford Solar Center. Moreover, the information collected in the local database is the starting point of future research that allows to understand and predict the space weather behavior. Likewise, our data can be correlated with data of the solar radiation intensity and observations in the optic and halpha bands carried out in the OAUTP, which can lead to a complete study of the solar activity. As the receiver discussed here meets the local conditions for Pereira-Colombia, it is possible to distribute it in schools to promote the scientific outreach, the development of other local projects in space weather and strengthening the radioastronomy in the country.

Finally, the SID monitor of the Universidad Tecnologica de Pereira can be modified in software and instrumentally in order to measure phase, which opens new possibilities for projects related with the measurement of phase such as the determination of the local dawn or the calculation of the height of the lowest layer of the ionosphere. This would be possible with the measurement and correlation of the signal reception times combined with mathematical and processing algorithms. This is also future work for the SID monitor developed at the OAUTP (Thomson and Rodger, 2005).
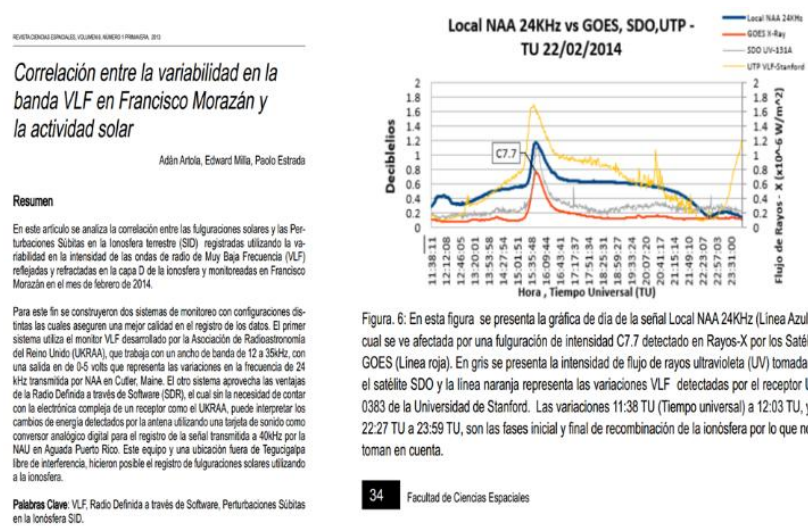

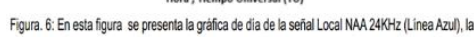

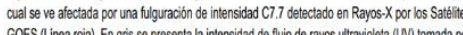
GOES (Linea a roal). En gins se presentat a lintensidad def flujo de rayos ultravideta (UV) tomada por

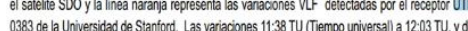

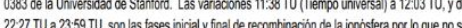
toman en cuenta-

34

Figure 9. Space weather project developed in the Universidad Francisco Morazán in El Salvador (Artola et al., 2017). 
El Sol Emite la Llamarada más Potente Registrada desde el 2006

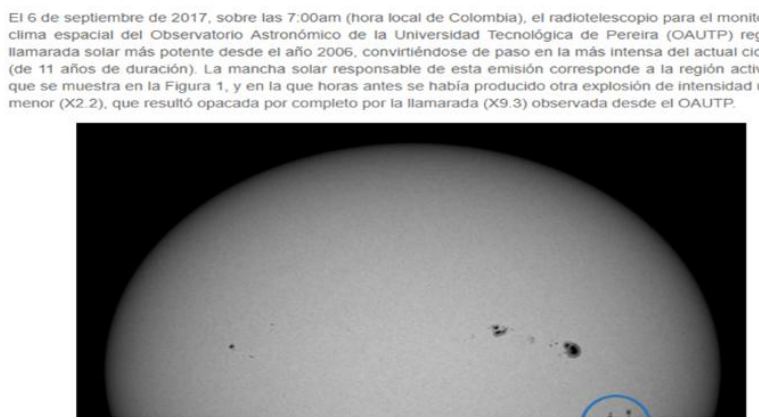

Figure 10. Press release written by OAUTP for scientific publication about space weather in Colombia.

\section{ACKNOWLEDGEMENTS}

The research group Alfa Orion, on behalf of the Astronomical Observatory, extend enormous gratitude to the Universidad Tecnológica de Pereira for providing the infrastructure and economic support for the development of this important project, as well as to the Faculty of Basic Sciences of the UTP, for the support in participation in academic events. Additionally, special thanks to the International Centre for Theoretical Physics (ICTP), the organizing committee of the International Space Weather Initiative (ISWI), and all of the affiliated sponsors for offering the full scholarship to participate and having the opportunity to present the results of this project with the international scientific community in the workshop held in the city of Trieste.

\section{REFERENCES}

Ammar, A. and Ghalila, H.: 2016, Acta Geophysica. 64 (6), 2794. Artola, A., Mills, E., \& Estrada, P.: 2013, Ciencias Espaciales. 6(1) 24.

Chakraborty S., Sasmal S., Basak T., Ghosh S., Palit S., Chakrabarti S. K. and Ray S.: 2017, Advances in Space Research. 60, 1787.

Grant R. A., Raulin J. P. and Freund F. T.: 2015, Physics and Chemistry of the Earth. 85, 69.

Inan U. S., Piddyachiy D., Peter W. B., Sauvaud J. A. and Parrot M.: 2007, Geophys. Res. Letter. 34, L07103.

Jaramillo, A. F., Galvis, H. D and Quintero, E. A.: 2017, Revista Ingeniería. 22(1), 83.

Kulkarni, P., Inan, U. S., Bell, T. F. and Bortnik, J.: 2008, J. Geophys. Res. 113, A07214.

Manoj, S. and Sundaram, S.: 2016, Journal of Chemical and Pharmaceutical Sciences. 9 (1), 548.

Petry, A., De Souza, J. R., De Campos, H. F., Pereira, A. G. and Bailey, G. J.: 2014, Advances in Space Research. 54, 22.

Quintero, E. A., Acuña, A. C., Puerta, L. M. and Pulgarín, O. E.: 2014, Revista De Investigaciones Universidad Del Quindio. 1 (26), 103.

Raulin J.-P., Matos David P. C., Hadano R., Saraiva A. C. V., Correia E and Kaufmann P.: 2009, Earth, Moon, and Planets. 104, 247.

Raulin J.-P., et al.: 2010, J. Geophys. Res. 115, A07301.

Sasmal S., Pal S. and Chakrabarti S. K.: 2014, Advances in Space Research. 54 (8), 1619.

Sharma, A. K., and More, C. T.: 2017, Research \& Reviews: Journal of Space Science \& Technology. 6(2), 1.

Shea, M. A. and Smart, D. F.: 1998, Advances in Space Research. 22(1), 29.

Tanaka Y. T., Raulin J. P., Bertoni F. C. P., Fagundes P. R., Chau J., Schuch N. J., Ha M., Hobara Y., Teresawa T. and Takahashi T.: 2010, The Astrophysical Journal Letters. 721, L24.

Thomson N. R., Rodger C. J. and Clilverd M. A.: 2005, Journal of Geophysical Research. 110, A06306. 